\title{
Energy Levels of Weak Coupling Magneto-Optical Polaron and Temperature Effect in Spherical Quantum Dot
}

\author{
Nsangou Issofa ${ }^{1}$, Alain Jerve Fotue ${ }^{1,}{ }^{*}$, Sadem Christian Kenfack ${ }^{1}$, Maurice Tiotsop ${ }^{1}$, \\ Michel Pascal Tabue Djemmo ${ }^{1,3}$, Amos Veyongni Wirngo ${ }^{1}$, Hilaire Fotsin ${ }^{2}$, Lukong Cornelius Fai ${ }^{1}$ \\ ${ }^{1}$ Mesoscopic and Multilayers Structures Laboratory, Department of Physics, Faculty of Science, University of Dschang, Dschang, Cameroon \\ ${ }^{2}$ Laboratory of Electronics and Signal Processing, Department of Physics, Faculty of Science, University of Dschang, Dschang, Cameroon \\ ${ }^{3}$ Laboratory of Mechanics and Modeling of Physical Systems, Faculty of Science, University of Dschang, Dschang, Cameroon
}

Email address:

fotuea@yahoo.fr (A. J. Fotue)

\section{To cite this article:}

Nsangou Issofa, Alain Jerve Fotue, Sadem Christian Kenfack, Maurice Tiotsop, Michel Pascal Tabue Djemmo, Amos Veyongni Wirngo, Hilaire Fotsin, Lukong Cornelius Fai. Energy Levels of Weak Coupling Magneto-Optical Polaron and Temperature Effect in Spherical Quantum Dot. American Journal of Modern Physics. Vol. 4, No. 4, 2015, pp. 158-164. doi: 10.11648/j.ajmp.20150404.12

\begin{abstract}
In this paper, we investigate the influence of the magnetic field and the temperature on the ground state energy of a weak coupling polaron in a spherical semiconductor quantum dot (QD) using the modified LLP method. The ground state energy of a weak coupling magneto-optical polaron is split into sub-energy levels and there is the degeneracy of the energy levels. It is also seen that the split energies are increasing functions of the electron-phonon coupling constant and decreasing functions of the magnetic field while the temperature is an increasing function of the cyclotron frequency for very low values of the longitudinal confinement length.
\end{abstract}

Keywords: Magnetic Field, Temperature, Modified LLP, Polaron Energy, Quantum Dot

\section{Introduction}

Due to the recent progress achieved in nanotechnology, it has become possible to fabricate low dimensional semiconductor structures. Special interest is being devoted to the quasi zero dimensional structures, usually referred to as quantum dots [1-9]. In such nanometer QD's, some novel physical phenomena and potential electronic device applications have generated a great deal of interest. This has laid a great challenge on theoretical physicists, that of developing the theory, based on the quantum mechanical regime. Recently, much effort [10-12] has been focused on exploring the polaron effect of QDs. Roussignol et al.[10] have shown experimentally and explained theoretically that the phonon broadening is very significant in very small semiconductor QDs. It has also been observed [11-12] that the polaron effect is more important if the dot sizes are reduced to a few nanometers. More recently, the related problem of an optical polaron bound to a Coulomb impurity in a QD has also been considered in the presence of a magnetic field. The theoretical investigation of the properties of the polaron has been done using the standard perturbation techniques [13], the variational Lee-Low-Pines method [1415] the modified LLP approach[16-17], the Feynman path integral method [18], numerical diagonalization [19] and by Green function methods [20]. The experimental data [21] showed, in particular, a large splitting width near the onephonon and two-phonon resonance in a InAs/GaAs QD. This was accounted for by the theoretical model via a numerical diagonalization of the Fröhlich interaction [19]. The required value of the Fröhlich constant was much larger (by a factor of two [19],) than that measured in bulk. In [18] using the Feynman path integral method, the authors observed that the quadratic dependence of the magnetopolaron energy is modulated by a logarithmic function and strongly depends on the Fröhlich electron-phonon coupling constant structure and cyclotron radius. Furthermore the effective electron-phonon coupling is enhanced by a high confinement and/or a strong magnetic field. In [21] the polaron energy in QDs was calculated using a Lee-Low-Pines (LLP) approach and it was found that the polaronic effect is more pronounced for small dot sizes. In [16], using a modified LLP approach, the number of phonons around the electron as well as the size of the polaron for the ground state and for the first two excited states is calculated via the adiabatic approach. It is important to note that, in the preceding works, the fact that the presence 
of magnetic field induces the interaction of the electron with the magnetic field and the precession of this electron along the $z-$ axis have not yet been taken into consideration. It is also instructive from the works presented above, to recall that polarons are often classified according to the Fröhlich electron-phonon coupling constant. Because it recovers simultaneously all types of coupling which characterize the Fröhlich electron-phonon coupling, the Feynman path integral method [18] has been seen as one of the best. The main feature of the method presented here is the modification of the LLP approach [16] by introducing two new parameters $b_{1}$ and $b_{2}$ in the traditional LLP approach, which permits us to obtain an "all coupling" polaron theory. Here the coupling is weak if $b_{1}=b_{2} \rightarrow 1$, strong coupling if $b_{1}=b_{2} \rightarrow 0$ and intermediate between these ranges.

In this work, we study the influence of the magnetic field and the total momentum on the polaron ground state energy. The article is organized as follows: in the second section, we describe the model and the Hamiltonian of the system and point out that, this Hamiltonian will be explicitly dependent on the Zeeman effect. In section 3, the analytical results of the ground state energy and of the polaron effective mass are obtained. In section 4, we present a brief result on the effect of temperature on the polaron, in section 5 , we discuss our results and, then, we end with the conclusion.

\section{Theory and Calculation}

The model we use consists of an electron confined in an isotropic potential box with tunable dimensions immersed in the field of the bulk longitudinal optical (LO)-phonon modes and interacting with an external magnetic field while the total momentum is directed along the z-axis. The dimensionless Hamiltonian describing the problem in Fröhlich units is given by [22]

$$
H=H_{e}+H_{p h}+H_{e-p h}
$$

$H_{e}$ represents the electronic Hamiltonian and is given by

$$
H_{e}=\frac{p^{2}}{2 m}+\frac{1}{2} m \Omega^{2} \rho^{2}+\frac{1}{2} m \omega_{2}^{2} z^{2}-\mu_{B} B_{e x t} g_{J} m_{J}
$$

where $p$ is the momentum, $\omega_{1}$ and $\omega_{2}$ measure the confinement in the $x y$ - plane and the $z$ - direction respectively and $\Omega^{2}=\omega_{1}^{2}+\omega_{c}^{2}$. The fourth term of the Hamiltonian is the interaction of the magnetic moment with the total angular momentum $J$; where $\mu_{B}$ is the Bohr Magneton, $g_{J}$ is the Landé $g$ factor, $m_{J}$ is the magnetic total angular momentum with numbers ranging from $-J$ to $J$. The Landé factor is given by $g_{J}=\frac{3}{2}+\frac{S(S+1)-L(L+1)}{2 J(J+1)}$; $S$ is the spin and $L$ is the angular momentum. $H_{p h}$ is the phonon Hamiltonian defined as

$$
H_{p h}=\sum_{Q} a_{Q}^{+} a_{Q}
$$

Where $a_{Q}^{+}\left(a_{Q}\right)$ are the creation (annihilation) operators for LO phonons of the wave vector $Q=\left(q, q_{z}\right), H_{e-p h}$ represents the electron-phonon Hamiltonian and is given by

$$
H_{e-p h}=\sum_{Q} V_{Q}\left[a_{Q} e^{i Q . r}+a_{Q}^{+} e^{-i Q . r}\right]
$$

$V_{Q}$ and $\alpha$ are the amplitude of the electron-phonon interaction and the coupling constant respectively given by

$$
\begin{gathered}
V_{q}=i\left(\frac{\hbar \omega_{L O}}{q}\right)\left(\frac{\hbar}{2 m \omega_{L O}}\right)^{1 / 4}\left(\frac{4 \pi \alpha}{V}\right)^{1 / 2} \\
\alpha=\left(\frac{e^{2}}{2 \hbar \omega_{L O}}\right)\left(\frac{2 m \omega_{L O}}{\hbar}\right)^{1 / 2}
\end{gathered}
$$

\section{Ground State Energy}

Adopting the mixed-coupling approximation of [23] we propose a modification to the first Lee-Low-Pines (LLP)transformation by inserting two variational parameters $b_{1}$ and $b_{2}$.

Our new unitary transformation is now

$$
U_{1}=\exp \left[i\left[\left(P_{\rho}-P_{\rho}\right) \rho b_{1}+\left(P_{z}-P_{\rho}\right) z b_{2}\right]\right]
$$

With

$$
P=p+\sum_{Q} a_{Q}^{+} a_{Q}
$$

being the total momentum of the polaron while

$$
P=\sum_{Q} Q a_{Q}^{+} a_{Q}
$$

is the momentum of the phonon.

The two new variational parameters are supposed to trace the problem from the strong coupling case to the weak coupling limit and to interpolate between all possible geometries.

The second transformation is of the form [1]

$$
U_{2}=\sum_{Q} u_{Q}\left(a_{Q}^{+}-a_{Q}\right)
$$

where $u_{Q}$ is a variational function. This transformation is called the displaced oscillator which is related to the phonon operators via the phonon wave vector through the relation

$$
\phi_{p h}=U_{2}\left|0_{p h}\right\rangle
$$

where $\left|0_{p h}\right\rangle$ is the phonon vacuum state since at low 
temperature there will be no effective phonons.

obtain

Applying the transformation in (3.1) on the Hamiltonian, we

$$
\begin{aligned}
H^{(1)}= & U_{1}^{-1} H U_{1}=\frac{p^{2}}{2 m}+\frac{1}{2} m \Omega^{2} \rho^{2}+\frac{1}{2} m \omega_{2}^{2} z^{2}+\mu_{B} B_{e x t} g_{J} m_{J}+b_{1}^{2}\left(P_{\rho}-P_{\rho}\right)^{2}+ \\
& +2 b_{1} p_{\rho}\left(P_{\rho}-P_{\rho}\right)+b_{2}^{2}\left(P_{z}-P_{z}\right)^{2}+2 b_{2} p_{z}\left(P_{z}-P_{z}\right)+\sum_{Q} a_{Q}^{+} a_{Q}+\sum_{Q} V_{Q}\left[a_{Q} e^{-i\left(b_{1} q . \rho+b_{2} q_{z} z\right)} e^{i Q . r}+a_{Q}^{+} e^{i\left(b_{1} q \rho+b_{2} q_{z} z\right)} e^{-i Q . r}\right]
\end{aligned}
$$

Applying the transformation (3.4) on (3.6) and expressing in Fröhlich units i.e. $2 m=\omega_{L O}=\hbar=1$, we obtain the ground state energy $\varepsilon_{g}$

$$
\begin{aligned}
\varepsilon_{g} & =\left\langle 0_{e}\left|-\nabla^{2}+\frac{1}{4} \Omega^{2} \rho^{2}+\frac{1}{4} \omega_{2}^{2} z^{2}+\mu_{B} B_{e x t} g_{J} m_{J}\right| 0_{e}\right\rangle+b_{1}^{2} P_{\rho}^{2}-2 b_{1}^{2} P_{\rho} P_{\rho}^{(0)}+b_{1}^{2}\left(P_{\rho}^{(0)}\right)^{2}+ \\
& +\sum_{Q} u_{Q}^{2}\left(1+b_{1}^{2} q^{2}+b_{2}^{2} q_{z}^{2}\right)+\left\langle 0_{e}\left|\left\langle 0_{p h}\left|2 b_{1} p_{\rho}\left(P_{\rho}-P_{\rho}+P_{\rho}^{(1)}-P_{\rho}^{(0)}\right)\right| 0_{p h}\right\rangle\right| 0_{e}\right\rangle+ \\
& +\sum_{Q} V_{Q} u_{Q}\left\langle 0_{e}\left|\left(\exp \left[-i\left(b_{1} q \cdot \rho+b_{2} q_{z} z\right)\right] \exp (i Q \cdot r)-\exp \left[i\left(b_{1} q \cdot \rho+b_{2} q_{z} z\right)\right] \exp (-i Q . r)\right)\right| 0_{e}\right\rangle+ \\
& +b_{2}^{2} P_{z}^{2}-2 b_{2}^{2} P_{z} P_{z}^{(0)}+b_{2}^{2}\left(P_{\rho}^{(0)}\right)^{2}+\left\langle 0_{e}\left|\left\langle 0_{p h}\left|2 b_{2} p_{z}\left(P_{z}-P_{z}+P_{z}^{(1)}-P_{z}^{(0)}\right)\right| 0_{p h}\right\rangle\right| 0_{e}\right\rangle
\end{aligned}
$$

where

$$
P^{(1)}=\sum_{Q} Q u_{Q}\left(a_{Q}+a_{Q}^{+}\right)
$$

and

$$
P^{(0)}=\sum_{Q} Q u_{Q}^{2}
$$

To evaluate this expression, we introduce the linear combination operators of the position and momentum of the electron by the following relation:

$$
\begin{aligned}
& p_{\mu}=\sqrt{\frac{m \hbar \lambda_{1}}{2}}\left(\sigma_{\mu}+\sigma_{\mu}^{+}\right) \\
& x_{\mu}=i \sqrt{\frac{\hbar}{2 m \lambda_{2}}}\left(\sigma_{\mu}-\sigma_{\mu}^{+}\right) \\
& p_{z}=\sqrt{\frac{m \hbar \lambda_{1}}{2}}\left(\sigma_{z}+\sigma_{z}^{+}\right) \\
& x_{\mu}=-i \sqrt{\frac{\hbar}{2 m \lambda_{2}}}\left(\sigma_{z}-\sigma_{z}^{+}\right)
\end{aligned}
$$

where the index $\mu$ refers to the $x$ and $y$ directions, $\lambda_{1}$ and $\lambda_{2}$ are variational parameters, while $\sigma$ and $\sigma^{+}$are respectively the annihilation and creation operators for the electron. Using the following commutator, $\left[x_{\mu}, p_{v}\right]=i \hbar \delta_{\mu \nu}$ and performing the required calculations, we may write the ground state energy as:

$$
\begin{aligned}
\varepsilon_{g} & =\frac{\lambda_{1}}{2}+\frac{\lambda_{2}}{4}+\frac{\Omega^{2}}{2 \lambda_{1}}+\frac{\omega_{2}^{2}}{4 \lambda_{2}}+\mu_{B} B_{e x t} g_{J} m_{J}+b_{1}^{2} P_{\rho}^{2}-2 b_{1}^{2} P_{\rho} P_{\rho}^{(0)}+b_{1}^{2}\left(P_{\rho}^{(0)}\right)^{2}+ \\
& +\sum_{Q} u_{Q}^{2}\left(1+b_{1}^{2} q^{2}+b_{2}^{2} q_{z}^{2}\right)+b_{2}^{2} P_{z}^{2}-2 b_{2}^{2} P_{z} P_{z}^{(0)}+b_{2}^{2}\left(\boldsymbol{P}_{z}^{(0)}\right)^{2}-2 \sum_{Q} V_{Q} u_{Q} S_{Q}
\end{aligned}
$$

with

$$
S_{Q}=\left\langle 0_{e}\left|\exp \left[ \pm i\left(b_{1} q . \rho+b_{2} q_{z} z\right)\right] \exp ( \pm i Q . r)\right| 0_{e}\right\rangle
$$

which can be rewritten as

$$
S_{Q}=\exp \left[-\left(1-b_{1}\right)^{2} \frac{q^{2}}{2 \lambda_{1}}\right] \exp \left[-\left(1-b_{2}\right)^{2} \frac{q_{z}^{2}}{2 \lambda_{2}}\right]
$$

Minimizing (3.11) with respect to the variational function $u_{Q}$ we obtain 


$$
\left[1+b_{1}^{2} q^{2}+b_{2}^{2} q_{z}^{2}+2 b_{1}^{2} q\left(P_{\rho}^{(0)}-P_{\rho}\right)+2 b_{2}^{2} q_{z}\left(P_{z}^{(0)}-P_{z}\right)\right] u_{Q}=V_{Q} S_{Q}
$$

Solving (3.14) with respect to $u_{Q}$, with the assumption that $\boldsymbol{P}^{(0)}$ differs from the total momentum by a scalar factor $\eta\left(P^{(0)}=\eta P\right)$, we get

$$
u_{Q}=\frac{V_{Q} S_{Q}}{1+b_{1}^{2} q^{2}+b_{2}^{2} q_{z}^{2}-2 b_{1}^{2} q P_{\rho}(1-\eta)-2 b_{2}^{2} q_{z} P_{z}(1-\eta)}
$$

Substituting (3.15) into (3.11) we obtain

$$
\begin{aligned}
\varepsilon_{g}= & \frac{\lambda_{1}}{2}+\frac{\lambda_{2}}{4}+\frac{\Omega^{2}}{2 \lambda_{1}}+\frac{\omega_{2}^{2}}{4 \lambda_{2}}+\mu_{B} B_{e x t} g_{J} m_{J}+b_{1}^{2} P_{\rho}^{2}(1-\eta)^{2}+b_{2}^{2} P_{z}^{2}(1-\eta)^{2}+ \\
& +\sum_{Q} \frac{V_{Q}^{2} S_{Q}^{2}\left(1+b_{1}^{2} q^{2}+b_{2}^{2} q_{z}^{2}\right)}{\left[1+b_{1}^{2} q^{2}+b_{2}^{2} q_{z}^{2}-2 b_{1}^{2} q P_{\rho}(1-\eta)-2 b_{2}^{2} q_{z} P_{z}(1-\eta)\right]^{2}}-2 \sum_{Q} \frac{V_{Q}^{2} S_{Q}^{2}}{\left[1+b_{1}^{2} q^{2}+b_{2}^{2} q_{z}^{2}-2 b_{1}^{2} q P_{\rho}(1-\eta)-2 b_{2}^{2} q_{z} P_{z}(1-\eta)\right]}
\end{aligned}
$$

But $\varepsilon_{g}(P)$ may be well represented by the first two terms of a power series expansion in $P^{2}$ as in [23]

$$
\begin{aligned}
\varepsilon_{g}(P)=\varepsilon_{g}(0)+\beta \frac{P^{2}}{2}+0\left(P^{4}\right)+\ldots & \\
\varepsilon_{g} & =\frac{\lambda_{1}}{2}+\frac{\lambda_{2}}{4}+\frac{\Omega^{2}}{2 \lambda_{1}}+\frac{\omega_{2}^{2}}{4 \lambda_{2}}+\mu_{B} B_{\text {ext }} g_{J} m_{J}-\sum_{Q} \frac{V_{Q}^{2} S_{Q}^{2}}{\left[1+b_{1}^{2} q^{2}+b_{2}^{2} q_{z}^{2}\right]}
\end{aligned}
$$
energy

Substituting for $S_{Q}$ in (3.13), the ground state energy in (3.18) finally becomes

$$
\varepsilon_{g}=\frac{\lambda_{1}}{2}+\frac{\lambda_{2}}{4}+\frac{\Omega^{2}}{2 \lambda_{1}}+\frac{\omega_{2}^{2}}{4 \lambda_{2}}+\mu_{B} B_{e x t} g_{J} m_{J}-\sum_{Q} \frac{V_{Q}^{2} \exp \left[-\left(1-b_{1}\right)^{2} \frac{q^{2}}{\lambda_{1}}\right] \exp \left[-\left(1-b_{2}\right)^{2} \frac{q_{z}^{2}}{\lambda_{2}}\right]}{\left[1+b_{1}^{2} q^{2}+b_{2}^{2} q_{z}^{2}\right]}
$$

which we rearrange as

$$
\varepsilon_{g}=E_{g}+\mu_{B} B_{\text {ext }} g_{J} m_{J}
$$

where

$$
E_{g}=\frac{\lambda_{1}}{2}+\frac{\lambda_{2}}{4}+\frac{\Omega^{2}}{2 \lambda_{1}}+\frac{\omega_{2}^{2}}{4 \lambda_{2}}-\sum_{Q} \frac{V_{Q}^{2} \exp \left[-\left(1-b_{1}\right)^{2} \frac{q^{2}}{\lambda_{1}}\right] \exp \left[-\left(1-b_{2}\right)^{2} \frac{q_{z}^{2}}{\lambda_{2}}\right]}{\left[1+b_{1}^{2} q^{2}+b_{2}^{2} q_{z}^{2}\right]}
$$

Under the influence of the magnetic field along the $z-$ direction with the polaron in the $1 S$ state, the angular momentum is $L=0$, the spin is $S= \pm \frac{1}{2}$ and the total momentum is $J=S+L=\frac{1}{2}$. These values show that the ground state split into four levels as given below:

$$
\begin{aligned}
& \varepsilon_{01} 1=E_{g}+2 \mu_{B} B_{e x t} \text { and } \varepsilon_{01} 2=E_{g}-2 \mu_{B} B_{e x t} \\
& \varepsilon_{02} 1=E_{g}+\frac{4}{3} \mu_{B} B_{e x t} \text { and } \varepsilon_{02} 2=E_{g}-\frac{4}{3} \mu_{B} B_{e x t}
\end{aligned}
$$

\section{Temperature Effect}

The polaron is no longer in the ground state entirely at a finite temperature. In such a case, the properties of the polaron are described by the statistical average of the phonon number. The average number of bulk LO phonons is given according to the quantum statistics theory as

$$
\bar{N}_{0}=\left[\exp \left(\frac{\varepsilon_{g}}{K_{B} T}\right)-1\right]^{-1}
$$

where $K_{B}$ is the Boltzmann constant and $T$ is the 
temperature of the system.

\section{Numerical Results and Discussions}

For the numerical results, we consider the weak coupling case, i.e. $b_{1}=b_{2}=1$. In this part, we display the plot of the ground state energy versus the electron-phonon coupling

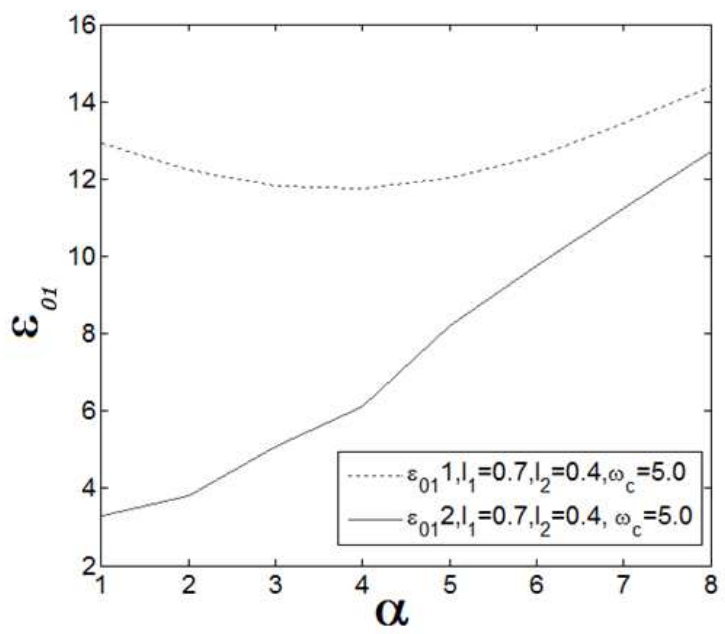

strength and the cyclotron frequency and that of the temperature versus the cyclotron frequency and the electronphonon coupling constant with the following polaron units: $R^{*}=\hbar \omega_{L O}$ and $r_{0}=\left(\hbar / 2 m^{*} \omega_{L O}\right)^{1 / 2}$

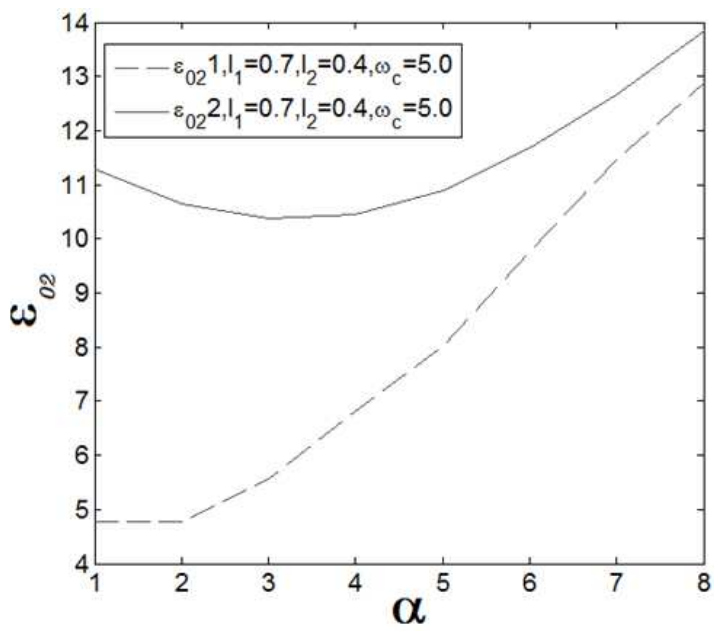

Figure 1. Split ground state energies of polaron in $1 S$ state as functions of coupling constant $\alpha$ with $l_{1}=0.7, l_{2}=0.4$ and $\omega_{c}=5.0$.
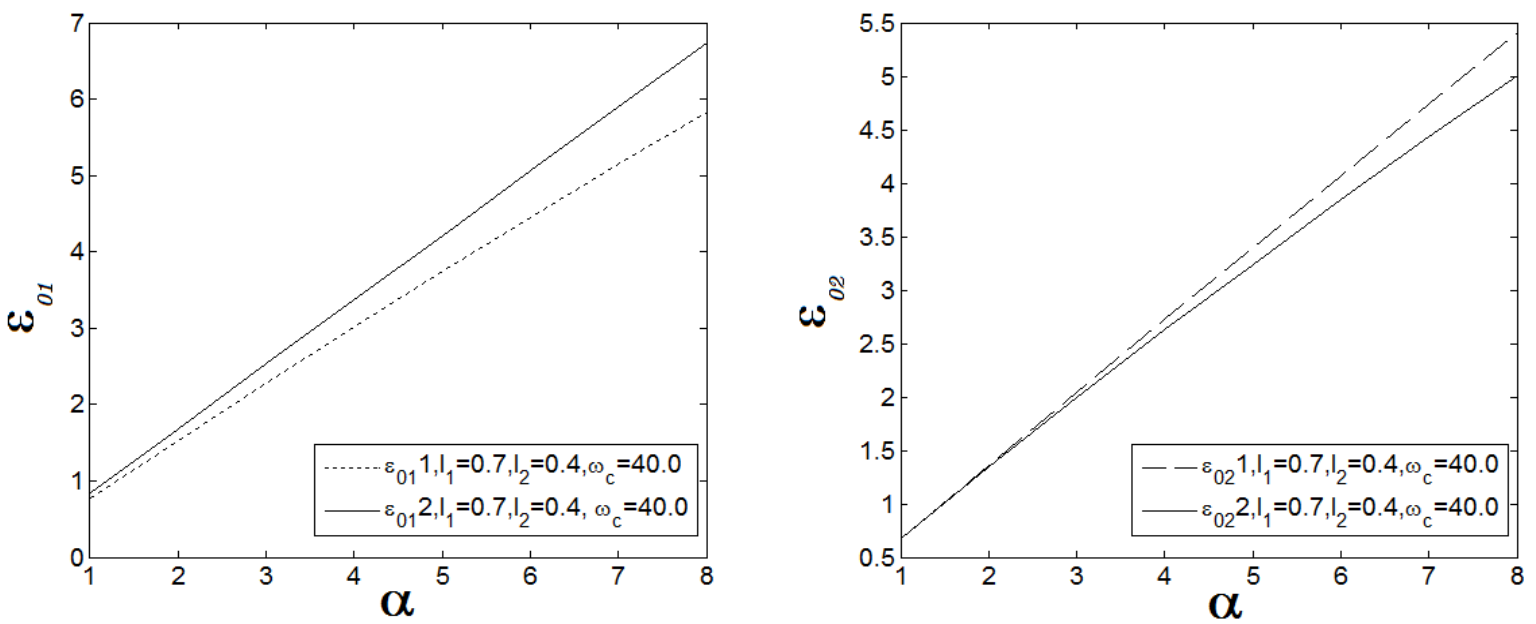

Figure 2. Split ground state energies of polaron in $1 S$ state as functions of coupling constant $\alpha$ with $l_{1}=0.7, l_{2}=0.4$ and $\omega_{c}=40.0$. 

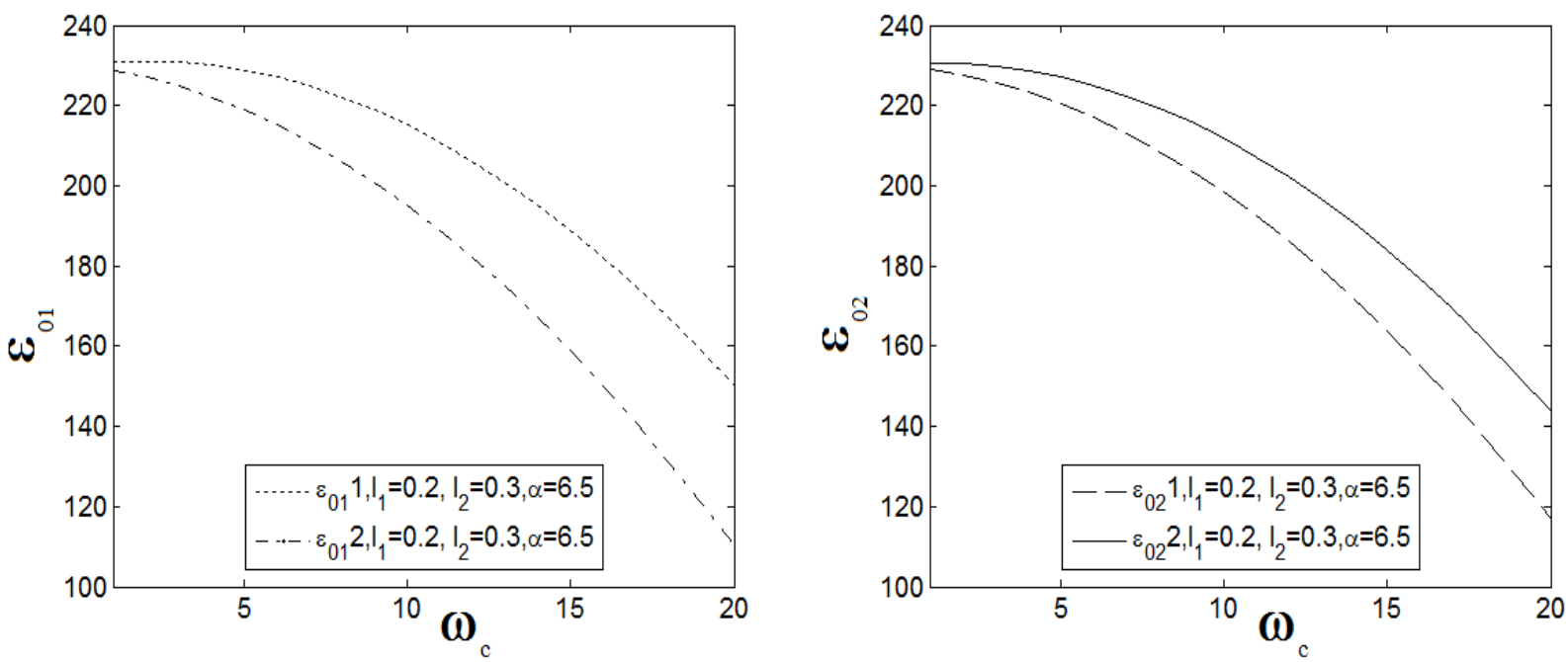

Figure 3. Split ground state energies of polaron in $1 S$ state as functions of cyclotron frequency $\omega_{c}$ with $l_{1}=0.2, l_{2}=0.3$ and $\alpha=6.5$.

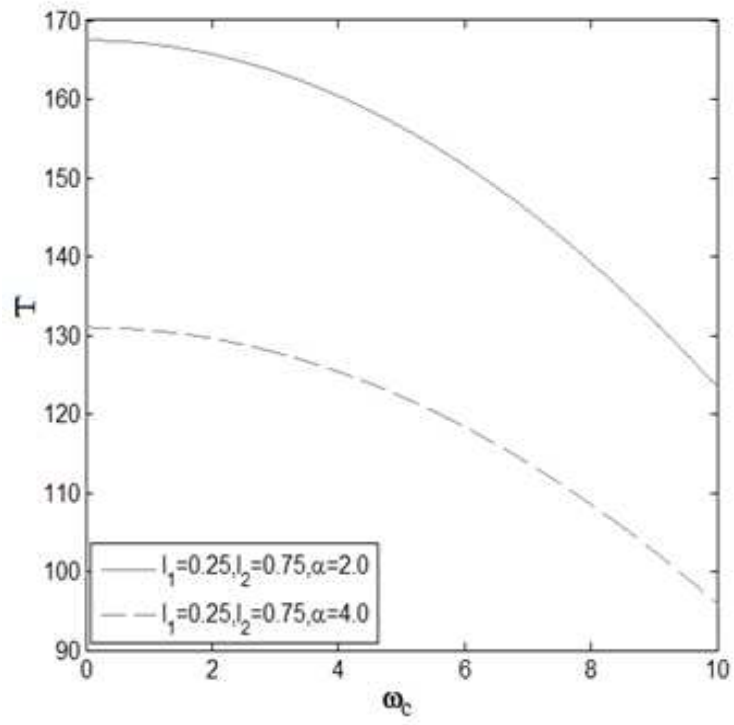

(a)

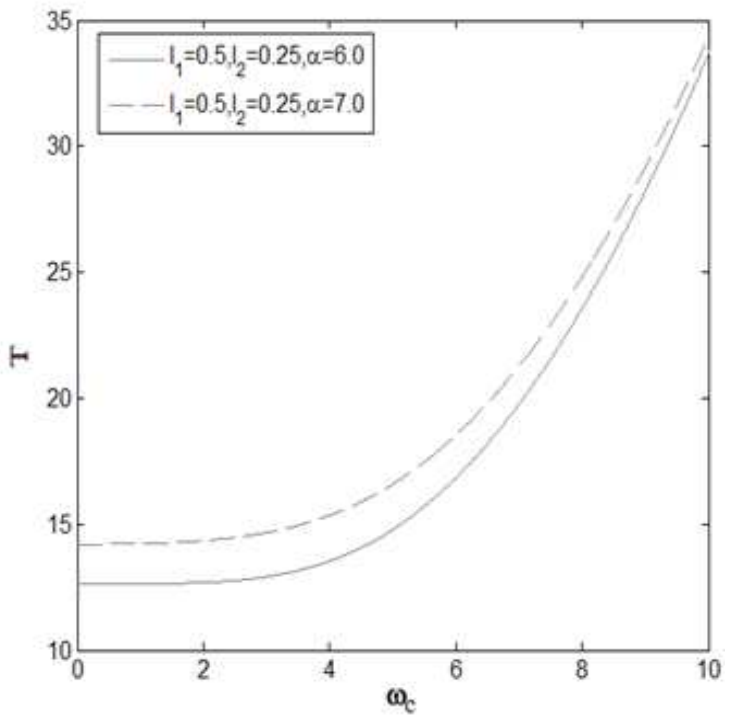

(b)

Figure 4. Temperature of polaron in $1 S$ state as function of cyclotron frequency $\omega_{c}:$ (a) $l_{1}=0.25, l_{2}=0.75$ (b) $l_{1}=0.5, l_{2}=0.25$.

In figures 1 and 2 we have plotted the energy of the polaron in the ground state as a function of the electron phonon coupling constant $\alpha$ for $l_{1}=0.7, l_{2}=0.4$ and $\omega_{c}=5.0$ (Fig. 1), $l_{1}=0.7, l_{2}=0.4$ and $\omega_{c}=40.0$ (Fig. 2). $\varepsilon_{01}$ and $\varepsilon_{02}$ are the energies of the polaron for $J_{J}=2$ and $g_{J}=\frac{4}{3}$ respectively. The split energies are increasing functions of the coupling constant $\alpha$. The increase of the electron-phonon coupling constant makes electrons to interact-with more phonons. This result is in agreement with that obtained by Shu-Ping Shan et al in [24-26]

In Figure 3, we have plotted the polaron energies as a function of the cyclotron frequency for $l_{1}=0.2, l_{2}=0.3$ and $\alpha=6.5$. From here, it is seen that the split energies are decreasing functions of the cyclotron frequency. As the magnetic field gets stronger, the electron moves away from the center and gets closer to the surface along the axis, resulting to a decrease in the contribution of the bulk LO phonon to the binding energy. This is very important in the control and modulation of the intensity of optoelectronic devices [27]

In figure 4 , we have plotted the temperature of the polaron as a function of cyclotron frequency for $l_{1}=0.25, l_{2}=0.75$

(Fig. 4a) and $l_{1}=0.5, l_{2}=0.25$ (Fig. $4 \mathrm{~b}$ ). From figure $4 \mathrm{a}$, it is seen that the temperature is a decreasing function of the cyclotron frequency for high values of the longitudinal confinement length. When the medium is large, it is normal that the temperature should decrease. In Fig. 4a, the temperature increases with the decrease of the longitudinal confinement length. The magnetic field is considered as a supplementary confinement to the electron and thus, the temperature has to increase when it increases (Fig. 4b). When the electron is highly confined in one direction, the motion in that direction becomes more important, thereby heating up the medium. These results are in in accordance with those 
obtained in [28-29].

\section{Conclusion}

In conclusion, with the use of the modified LLP method, we have studied the energy of a weak coupling polaron and the effect of temperature in an anisotropic QD subjected to a magnetic field and interacting with the momentum along the $z$ - direction. It is found that there is the splitting and degeneracy of the energy levels. We also see that the split energies are increasing functions of the coupling constant and decreasing functions of the cyclotron frequency. The enhancement of the coupling strength is very important in the construction of quantum computers since it leads to the conservation of its internal properties such as superposition states against the influence of its environment, which can induce the construction of coherent states and cause coherence quenching. So, we have more flexible and tunable methods than the parabolic and the asymmetrical QD to restrain quantum decoherence, for example, extending the effective confinement lengths appropriately and choosing polar materials with weaker coupling strength. We also see that, the temperature is a decreasing function of the cyclotron frequency at a certain value of the transverse confinement length. When the electron is more confined in the longitudinal direction, the temperature is an increasing function of the cyclotron frequency.

\section{References}

[1] R. T. Senger and A. Erçelebi. Solid State Phys, 22,169 (1998)

[2] Y.B. Yu., S.N.Zhu, K. X.Guo. Solid State Commun, 132 (10), 689 (2004)

[3] Liang, X.X., Gu, S.W., Lin, D.L. Phys. Rev. B., 34 (4), 2807 (1986)

[4] Zhu, K.D., Kobayashi, T. 92 (4), 353 (1994)

[5] Licari, J.J., Evrard, R. Phys. Rev. B., 15 (4), 2254 (1977)

[6] Das Sarma S. and Mason B.A. Ann. phy.NY,163 (1), 78 (1985)

[7] Licari J.J. Solid State Commun, 29 (8), 625 (1979)

[8] Comas F., Trallero-inner, C, Riera, R. Phys. Rev. B., 39(9), 5907 (1989)

[9] Yu Yi-Fu, Xiao Jing-Lin, Yin Ji-Wen and Wang Zi-Wu. Chinese Physics B., 17(6), 2236 (2007)
[10] P. Roussignol, D. Ricard and C. Flytzanis. Phys. Rev. Lett., 62, 312 (1989)

[11] K. D. Zhu and S. W. Gu. J. Phys.: Condens. Matter, 4, 1291 (1992)

[12] S. Mukhopadhyay and A. Chatterjee. J. Phys.: Condens. Matter, 8 (22), 4017 (1996)

[13] K. D. Zhu and S. W. Gu. Phys. Lett. A., 163 (5-6), 435 (1992)

[14] A. Chatterjee and S. Mukhopadhyay. Acta Phys. Polon. B., 32 (2), 473 (2001)

[15] S. Hameau, Y. Guldner, O. Verzelen, R. Ferreira, G. Bastard, J. Zeman, A. Lemaitre, and J. M. Gerard. Phys. Rev. Lett., 83 (20), 4152 (1999)

[16] ZherSamak, Bassam Saqqa. An - Najah Univ. J. Res. (N. Sc.), 23, 15 (2009)

[17] ZherSamak, BassamSaqqa. An - Najah Univ. J. Res. (N. Sc.), 24, 55 (2010)

[18] T. Stauber, R. Zimmermann, and H. Castella. Phys. Rev. B., 62 (11), $7336(2000)$

[19] M. Tchoffo , L .C. Fai, N.Issofa, S.C.Kenfack, J.T.Diffo, A. MODY. International Journal of Nanoscience 8 (4), 455 (2009)

[20] T. Inoshita, H. Sakaki. Phys. Rev. B., 46(11), 7260 (1992)

[21] Satyabrata, Sahoo. Phys. letters A., 238 (6), 390 (1998)

[22] Erçelebi A. and R. T. Senger, R.T. J. Phys.: Condens. Matter, 6(28), 5455 (1994)

[23] A. J. Fotue, S. C. Kenfack, H. Fotsin, M. Tiotsop, L. C. Fai and M. P. Tabue Djemmo physical science international journal, 6 (1), 25 (2015)

[24] Shu-Ping Shan, Shi-Hua Chen and Jing-Lin Xiao, QD J. low Temp Phys 176, 93(2014)

[25] Ji-Wen Yin, Wei -ping Li, Yi-Fu Yu and Jing-lin xiao, J. low Temp Phys (2011) 163, 53(2011)

[26] S.H. Chen, J. L. International Journal of Modern Physics B. $22(16), 2611(2008)$

[27] N. Kervan, T. Altanhan, A. Chatterjee. Phys. Lett. A. 315 (3-4), 280(2003)

[28] Jing-Lin Xiao, J. Low Temp Phys 168, 297(2012)

[29] Cui-Lan Zhao and Jing-Lin Xiao, J. Low Temp Phys 160, 209 (2010) 